\title{
Applications of minimally invasive cardiac output monitors
}

\author{
Jahan Porhomayon ${ }^{1,3^{*}}$, Gino Zadeii ${ }^{2}$, Samuel Congello ${ }^{2}$ and Nader D Nader ${ }^{1}$
}

\begin{abstract}
Because of the increasing age of the population, critical care and emergency medicine physicians have seen an increased number of critically ill patients over the last decade. Moreover, the trend of hospital closures in the United States $t$ imposes a burden of increased efficiency. Hence, the identification of devices that facilitate accurate but rapid assessments of hemodynamic parameters without the added burden of invasiveness becomes tantamount. The purpose of this review is to understand the applications and limitations of these new technologies.
\end{abstract}

\section{Review}

The ultimate goal of any hemodynamic monitoring system is to provide the clinicians with additional information on the underlying pathological condition and to guide fluid or vasopressor therapy. Cardiac output measurement and its response to therapeutic interventions are frequently used in critically ill patients. As the use of $\mathrm{CO}$ monitoring devices increases today, it is necessary to understand the application of such devices in different clinical settings. For many years pulmonary artery catheter (PAC) thermodilution cardiac output assessment was the monitor of choice for the management of critically ill patients. Thermodilution is a modification of the original indicator dilution techniques in which the injectate has a defined volume and temperature from which the thermodilution curve is generated [1]. As with the other indicator dilution techniques, $\mathrm{CO}$ is calculated from the area under the indicator thermodilution curve using the modified Stewart-Hamilton equation [2]. PAC was first used in dogs, and subsequently in humans 50 years later [2]. PAC provides valuable measurements, including right atrial pressure, right ventricular pressures, pulmonary artery pressures, pulmonary artery occlusive pressure, mixed venous saturation $\left(\mathrm{SvO}_{2}\right)$, and $\mathrm{CO}$. The derived hemodynamic variables are systemic and pulmonary vascular resistances. The major obstacle for the

\footnotetext{
* Correspondence: jahanpor@buffalo.edu

'VA Western New York Healthcare System, Division of Critical Care and Pain Medicine, Department of Anesthesiology, State University of New York at Buffalo School of Medicine and Biomedical Sciences, Buffalo, NY, USA Full list of author information is available at the end of the article
}

use of PAC has been the lack of demonstrating patient benefit and its level of invasiveness. Several prospective trials have demonstrated the lack of benefit from PACs. The PAC-man trial indicated that the routine placement of PACs had no effect on morbidity or mortality, and the ESCAPE trial found no difference in mortality or length of hospital stay when PAC parameters were compared with clinical assessment in the management of severe congestive heart failure patients [3-6]. Furthermore, for using PAC now, many physicians have lost the training, confidence, and familiarity with its use. PAC should probably be used only in selected patients by experienced practitioners. Contraindications to the insertion of PAC include tricuspid or pulmonary valve endocarditis/mechanical valve and right heart mass or thrombus. Like the PAC, each of these newer monitoring modalities requires education and training for effective use. For emergency room physicians, each technology provides a set of advantages and limitations. Minimally invasive cardiac output monitors allow for time efficiency in the emergency department setting and provide valuable information regarding the overall cardiovascular status of the patient. Declining cardiac index in trauma patients may indicate the need for revaluation of the patient. In general, early goal-directed therapy is usually better in the early phase of critical illness in contrast to late stages for sick patients. Minimally invasive monitoring devices for optimal $\mathrm{CO}$ and global oxygen balance may be of particular interest for emergency medicine physicians in the perioperative setting, acute lung injury, hypothermia induction, or preload and fluid

\section{实}


responsiveness assessment in the management of septic shock and acute respiratory distress syndrome. Currently the evidence and literature have not necessarily caught up with the trends in the US and Europe with these devices, and partts of the article represent the authors' experience.

\section{Minimally invasive CO monitors}

$\mathrm{CO}$ monitors use different principles for measuring $\mathrm{CO}$. They include Doppler technology, echocardiography, pulse contour analysis, transpulmonary thermodilution, bioimpedance, bioreactance and Fick's principle.

\section{Esophageal Doppler}

Esophageal Doppler (ED) measuring aortic blood flow velocity was first introduced in 1971 [7]. The ED monitor measures the velocity of blood flow in the descending thoracic aorta using a flexible ultrasound probe. When combined with the aortic cross-sectional area it allows measurement of stroke volume and CO. The aortic diameter is obtained from a built-in nomogram or by direct measurement using $\mathrm{M}$-mode echocardiography. A metaanalysis of several trials in critically ill patients showed high validity, but they were all performed in stable hemodynamic patients [8]. This meta-analysis suggested that $\mathrm{ED}$ was good at determining trends in $\mathrm{CO}$ but less effective in measuring absolute $\mathrm{CO}$. The main application of this device has been for preload optimization, myocardial contractibility and goal-directed fluid therapy for surgical patients (Figure 1) [9-11]. ED can be safely utilized in emergency departments. In the study by Rodriguez et al. [12], they demonstrated in a prospective manner that cardiac output evaluation using ED in the emergency room was superior to physician estimation of cardiac output. He concluded that esophageal Doppler measurement of $\mathrm{CO} / \mathrm{CI}$ appears to be practical from a physician time standpoint. Marquez et al. [13] demonstrated good correlation between ED and the LIDCO devices in cardiac surgery patients. Seoudi et al. prospectively investigated the correlation between PAC and ED in surgical trauma. On the basis of this study, it is reasonable to conclude that the ED is a valuable adjunct technology for $\mathrm{CO}$ and preload assessment for patients in the emergency medicine ward on mechanical ventilation, regardless of the level of mechanical ventilatory support [14]. The current literature supports the use of ED for assessment of cardiac output and left ventricular filling pressure [15-17]. This device is operator dependent, and placement of the probe in the wrong position can alter the $\mathrm{CO}$ reading. Additional limitations include the need for intubation of the trachea, expense, inaccurate $\mathrm{CO}$ in patients with aortic regurgitation, and the assumption that the division of the blood flow from the descending aorta is constant with even distribution to the brachiocephahlic

Velocity

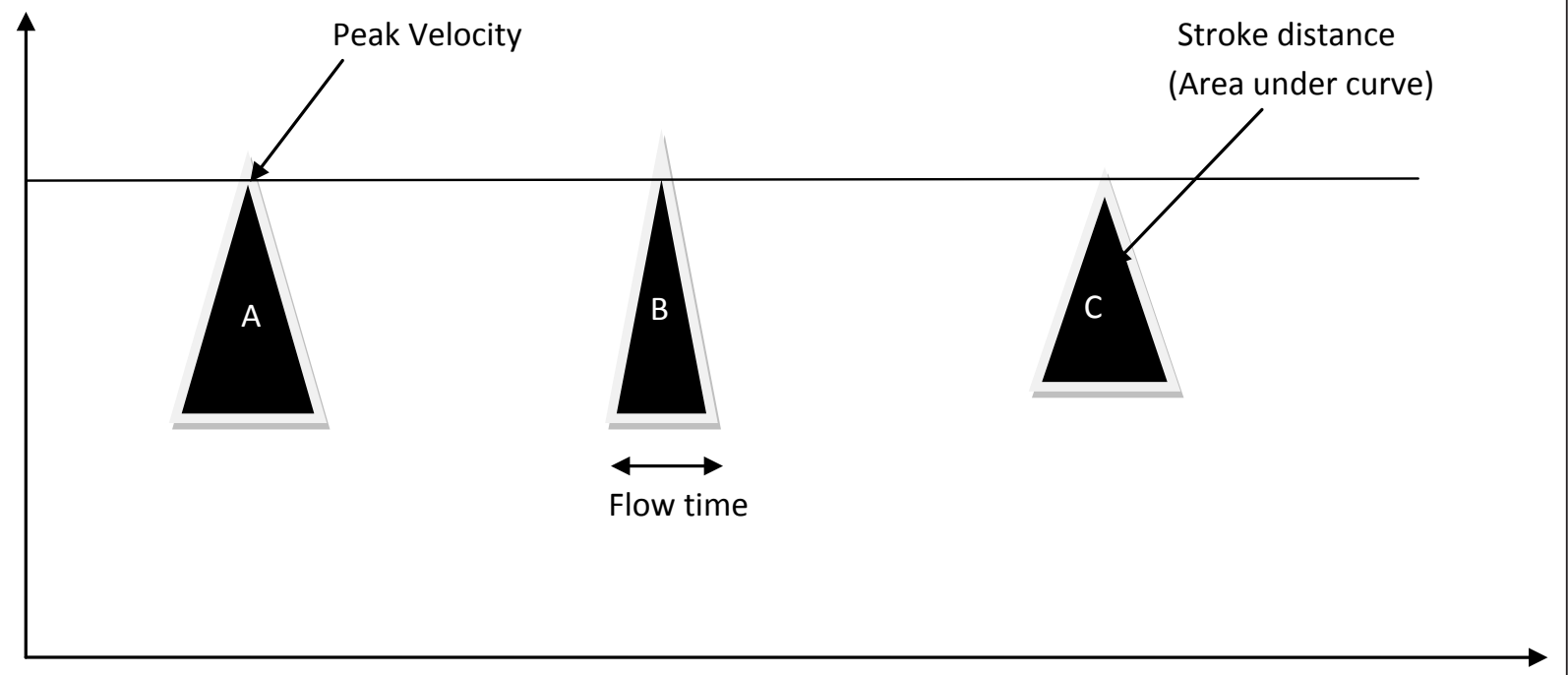

Time

Figure 1 Esophageal Doppler has the ability to measure flow time and peak velocity. The flow time is the time from the beginning of aortic pulse waveform upstroke to its return to baseline. A. Peak velocity is a good indicator of myocardial contractibility (normal). B. The left ventricular ejection time (or flow-time) corrected for heart rate provides an index of preload (hypovolemia). C. Left ventricular failure. Note that during hypovolemia and heart failure the stroke distance is decreased. 
and coronary arteries. This assumption is not always true in very ill patients.

\section{Echocardiography}

Echocardiography has been used in the ICU and emergency medicine for many years to diagnose the underlying cause of hemodynamic instability. Like ED, echocardiography uses Doppler technology, but it relies on direct visualization of the cardiac anatomy and flow dynamics. The American College of Emergency Physicians encourages emergency medicine physicians to be able to rapidly diagnose pericardial tamponade and electromechanical dissociation, which represent truly emergent and potentially lethal cardiovascular conditions. A focused point of care exam will enable the emergency medicine physician to quickly assess: hemodynamic states [18] and unexplained hypotension [19], congestive heart failure, pericardial effusion, and pulmonary emboli [20]. In addition, it provides an emergency medicine physician an important tool to assess the efficiency of resuscitation and ultimately improve patient outcome [21]. Moreover, if the causes of circulatory failure are obscure, echocardiography provides the ability to evaluate structural abnormalities such as:

1. Wall motion abnormality for the diagnosis of myocardial injury [22] and evaluation of cardiac preload by estimating inferior vena cava (IVC) collapsibility [23].

2. Ventricular systolic dysfunction [24] and cardiac output (Figure 2).

One potential disadvantage of this device is the additional training required to make a proper diagnosis. Detailed cardiac echocardiography for diagnosis of complex cardiac diseases requires more advance training. Echocardiography measures $\mathrm{CO}$ only in a single point in time and is not suitable for trend analysis.

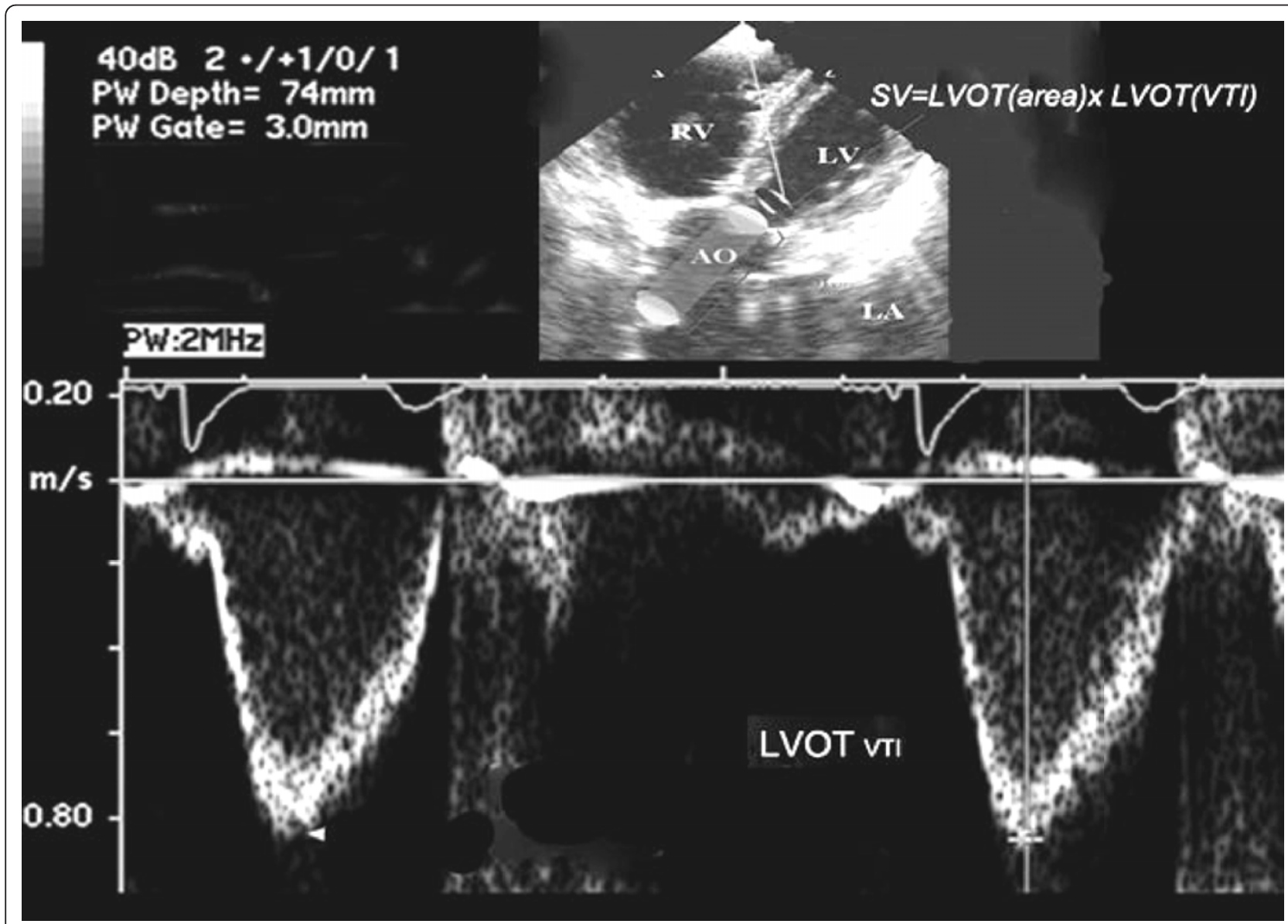

Figure 2 Blood leaves the left ventricular outflow tract (LVOT) as a cylinder (aorta shown in red). The volume of the cylinder is equal to the stroke volume (SV). In order to calculate SV, the echocardiographer must obtain the diameter of the LVOT and velocity time integral (VTI) of the blood measured at the same exact location. $A O=$ Aorta, $L V=$ left ventricle, $R V=$ right ventricle, $L A=$ left atrium. 


\section{Pulse contour analysis}

Pulse contour analysis of cardiac output is based on the principles that stroke volume can be continuously estimated by analyzing the arterial pressure waveform obtained from an arterial line. The origin of the pulse contour method for estimation of the beat-to-beat stroke volume is based on the Windkessel model described by Otto Frank in 1899. In 1974, Wesseling et al. developed an algorithm that can be used to monitor stroke volume (SV) [25]. CO is calculated from the area under the curve of the systolic portion of the arterial pressure waveform divided by the aortic impedance multiplied by the heart rate. Currently, there are different commercially available devices that measure $\mathrm{CO}$ based on the pulse contour analysis method.

The most frequently used ones are the calibrated PiCCO monitor system (PULSION Medical Systems, Munich, Germany), the LiDCO monitoring system (LiDCO Ltd., London, UK), which is available as either a calibrated (LiDCOplus) or uncalibrated device (LiDCOrapid), and the uncalibrated FloTrac/Vigileo device (Edwards Life Sciences, Irvine, CA). The pulse contour devices are utilized with greater frequency in our local practice; hence, they are covered in more detail.

\section{PiCCO system}

The PiCCO system uses the pulse contour method based on the Wesseling algorithm for the calculation of $\mathrm{CO}$. The system is periodically calibrated via the thermodilution method to calibrate the pulse pressure algorithm. PiCCO is a cardiac monitor that measures cardiac output and several volumes such as intrathoracic blood volume (ITBV), global end diastolic volume
(GEDV), and extra vascular lung water (EVLW) [26]. $\mathrm{PiCCO}$ can also provide pulse contour parameters, which consist of continuous $\mathrm{CO}$, systemic vascular resistance (SVR), stroke volume variation (SVV), and pulse pressure variation. The PiCCO system requires a thermistor-tipped central venous catheter and an arterial line usually introduced via the femoral, axillary, or brachial artery. After central venous injection of the cold indicator, the thermistor in the tip of the arterial catheter measures the downstream temperature changes. The $\mathrm{CO}$ is then calculated by analysis of the thermodilution curve using a modified Stewart-Hamilton algorithm. Pulse contour analysis continuously measures stroke volume and arterial pressure. $\mathrm{CO}$ and systemic vascular resistance (SVR) are calculated (Figure 3). Different studies in a variety of clinical settings have been performed in recent years validating the $\mathrm{PiCCO}$ system against intermittent pulmonary artery thermodilution (ITD) $[27,28]$. Goal-directed therapy with this technology has been reported in patients undergoing CABG surgery [29] and for preload optimization [30,31]. In the study by Uchino et al., the use of PiCCO was associated with a greater positive fluid balance and fewer ventilator-free days. After correction for confounding factors, the choice of monitoring did not influence major outcomes, whereas a positive fluid balance was a significant independent predictor of outcome [32]. This device has mainly been utilized in critical care setting but

PiCCO has the ability to measure (ITBV), (EVLW), and cardiac function index (CFI). These parameters are of interest as they are considered to be the most specific measures of cardiac preload, pulmonary edema, contractility, and a global indicator of cardiac performance.

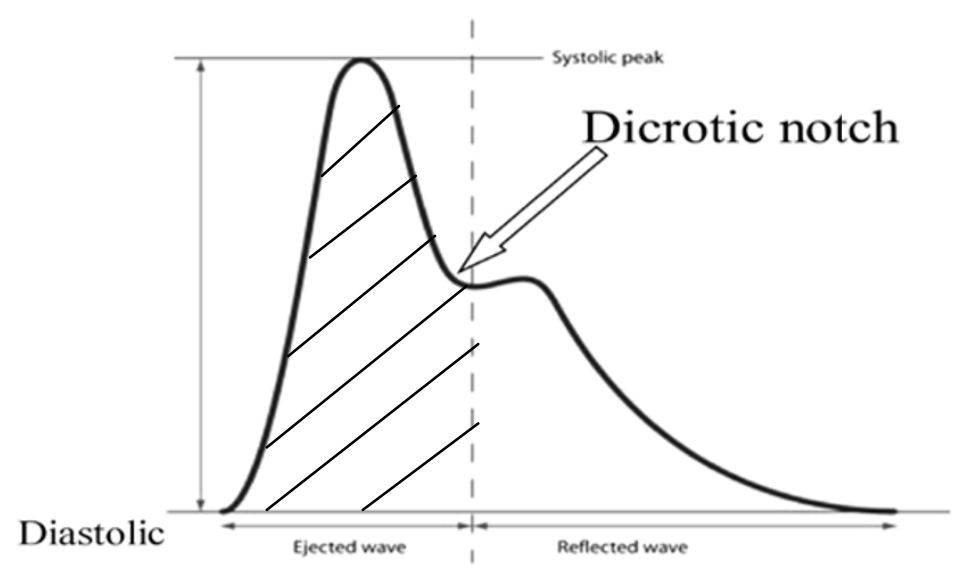

(Systolic Phase)

(Diastolic Phase)

Figure 3 PiCCO system: Stroke volume is the area under systolic portion of arterial pulse waveform (shaded). Stroke volume is calculated $=$ [area under systolic phase (shaded) + aortic compliance $] \times$ shape of pressure curve. 
Therefore, PiCCO may give an emergency medicine physician a powerful tool for managing critically ill patients [29].

\section{Flo-Trac}

Flo-Trac is another pulse contour $\mathrm{CO}$ monitoring system (Vigileo, Edwards Life Sciences) that was introduced in 2005. A special blood flow sensor, which is connected to an arterial line (radial, brachial, axillary or femoral artery), is needed. No external calibration is necessary [33-35]. This device calculates $\mathrm{CO}$ on a continuous basis by multiplying the pulse rate by calculated stroke volume. The direct relationship between arterial pulsatility and the stroke volume is used to calculate CO. Based on the model described by Langewouters et al. [36] individual demographics (body surface area, age, gender) are used for estimation of aortic compliance. Vascular compliance and resistance are determined using arterial waveform analysis. Several studies $[33,37,38]$ have been performed concerning the accuracy of Vigileo $\mathrm{CO}$ monitoring in a variety of patients with different software versions of the device. The Vigileo monitor has been used in emergency medicine setting for resuscitation of burn victims with good result [39]. Stroke volume variation on this monitor can help clinicians to assess fluid responsiveness in the initial phase of septic shock. However, rapid changes in vascular motor tone may lead to impaired accuracy of $\mathrm{CO}$ monitoring. Flow-Trac accuracy is worse with arterial wave artifact, compromise of the arterial catheter, aortic regurgitation, intense peripheral vasoconstriction, irregular pulse, and severe cardiac hypo-function. Thus, its reliability is influenced by various conditions, especially in critically ill patients [40].

\section{Lithium dilution $\mathrm{CO}$}

The technique of using lithium dilution to measure $\mathrm{CO}$ was first described in 1993 by Linton et al. [41]. This technique uses pulse contour analysis for $\mathrm{CO}$ measurement and lithium dilution for system calibration. A small dose of lithium is injected into a peripheral vein, and an ion selective electrode is attached to a peripheral arterial line. The area under the curve of a plot of lithium concentration against time allows accurate calculation of the $\mathrm{CO}$. The lithium dilution technique is of sufficient accuracy when there is constant blood flow and uniform mixing of blood. There are advantages and limitations to this technology, discussed in detail by Pearse et al. [42]. Other groups have demonstrated the accuracy of LIDCO [43]. Costa et al. showed good agreement among the LiDCO, PAC, and PICCO systems [44]. The major limitation to this device is the need for repetitive blood draws and, in the presence of neuromuscular blocking drugs, interference with calibration.

\section{Bioimpedance}

The use of the electrical signal across the thorax to measure CO goes back to early 1970 [45]. Bioimpedance technology uses electrical resistance charges across the chest for identification of cyclic changes in blood flow. $\mathrm{CO}$ is then continuously estimated by analyzing beat-tobeat signal variation. $\mathrm{CO}$ calculation is based on different mathematical models. Early studies demonstrated only a fair correlation between thoracic electrical impedance (TEB) and thermodilution CO [46]. Despite many adjustments of the mathematical algorithms, validation studies continue to show mixed results [47]. In addition, TEB accuracy decreases with excessive lung water and pulmonary edema [48]. In recent years advances in bioimpedence technology have resulted in development of a novel technology placing the electrodes on endotracheal tube cuff (ECOM, CONMED, Utica, NY, USA). The proximity of the ascending aorta and trachea facilitated the design of this device. It can optimize the current delivery and signal recording from changes in the ascending aorta [49]. Because the tracheal mucosa produces mucous and fluid, the endotracheal cardiac output monitor (ECOM) electrodes have been designed to reduce the effects of fluid and mucous buildup. This device appears promising, and preliminary data indicate adequate reliability of this device [49-51]. The main disadvantage of ECOM is the need for an endotracheal tube and mechanical ventilation. ECOM can be used in emergency medicine for preload assessment and response to vasopressor therapy.

\section{Bioreactance}

Bioreactance (NICOM; Cheetah Medical, Vancouver, WA, USA) is a unique technology for assessment of cardiac output [52]. It is similar to bioimpedance in that electrical current is applied to the chest via two leads. The bioreactance technique analyzes the frequency variations of the delivered oscillating current. This will result in a higher signal-to-noise ratio, and thus result in improved performance of the device. There have been several studies comparing the NICOM monitor to other monitors (PiCCO, Flo-Trac, PAC). These studies showed high agreement with the various monitors [52-56]. This device has very few known disadvantages and can be used in variety of settings.

\section{Fick's principle}

Adolf Fick spent most of his life studying muscle metabolism, but in a brief publication in 1870, he described how mass balance might be used to measure cardiac output [57]. Later Guyton et al quoted the original work and expanded upon it [58]. It is based on the conservation of mass, such that the total uptake or release of a substance by an organ is the product of the blood flow 
to that organ multiplied by the arteriovenous concentration difference [59]. CO by modified Fick's method measures carbon dioxide $\left(\mathrm{CO}_{2}\right)$ production and exhaled or end tidal $\mathrm{CO}_{2}$ at baseline and during a brief period of rebreathing. This will allow calculation of pulmonary artery blood flow. A new monitor called the NICO system (Novametrix Medical Systems, Wallingford, CT) uses Fick's equation for $\mathrm{CO}_{2}$ elimination. It is relatively noninvasive. Its principle states that over a fixed period of time the amount of $\mathrm{CO}_{2}$ leaving the lungs in the arterial blood is equal to the amount brought into the lungs in the venous blood minus the amount eliminated through the lungs. With this method, the $\mathrm{CO}$ is computed on breath-by-breath measurements of $\mathrm{CO}_{2}$ elimination. $\mathrm{CO}$ is proportional to the change in $\mathrm{CO}_{2}$ elimination divided by the change in end tidal $\mathrm{CO}_{2}$ resulting from a brief rebreathing period. Rebreathing measurements are made every $3 \mathrm{~min}$ for $35 \mathrm{~s}$. The main drawback to this system is the assumption about the shunt fraction and arterial $\mathrm{CO}_{2}$ being equal to end-tidal $\mathrm{CO}_{2}$. Clinical and experimental data for $\mathrm{CO}$ determinations with the NICO monitor [60] give a better approximation of $\mathrm{CO}$ in patients who are less critically ill and have normal alveolar gas exchange. Additionally, preload optimization may be difficult using the NICO monitor, and caution should be exercised before using this monitor for fluid administration. Potentially large volumes of fluid may be administered to achieve desired endpoints [61]. Advantages of NICO include the easy setup and providing capillary blood flow and ventilator parameters, such as the ratio of tidal volume to dead space. The NICO monitor assumes that the partial venous $\mathrm{CO}_{2}$ concentration reflects the level of $\mathrm{CO}_{2}$ stored in the body. Therefore, any changes in metabolism or ventilation may alter the reliability of this monitor. Pulmonary shunting and heterogeneous ventilation decrease the

Table 1 Cardiac output monitors

\begin{tabular}{lll}
\hline & Advantages & Disadvantages \\
\hline PAC & Measure CVP & Pulmonary infarction \\
& Intermittent and continuous & Rupture of pulmonary artery \\
& SVR can be obtained & Arrhythmias \\
\hline Pulse wave & Need right heart catheterization
\end{tabular}

Pulse wave

Analysis

A. PICCO

Intermittent and continuous

Need a central venous access

Measures GEDV/EVLW

Estimate preload

B. LIDCO

Intermittent and continuous

SVR can be obtained

\begin{tabular}{ll}
\hline C. Flo-trac & SVR can be obtained \\
& Measure PPV/SW \\
& Many validation studies \\
\hline Esophageal Doppler & Less invasive \\
& Simple to use
\end{tabular}

\begin{tabular}{ll}
\hline Echocardiography & Provides detailed cardiac information \\
& Estimate preload
\end{tabular}

\begin{tabular}{lll}
\hline Bioreactance & Non-invasive & Numerous mathematical assumptions \\
& Continuous & Signal stability fails after $24 \mathrm{~h}$ \\
\hline Bioimpedence & Continuous & Numerous mathematical assumptions \\
& Difficult to set up & Signal stability fails after $24 \mathrm{~h}$ \\
\hline Flick's Principle & Easy set up & Not suitable for unstable patient \\
& Provides additional ventilatory parameters & Shunt can affect CO estimation \\
\hline
\end{tabular}

CVP central venous pressure, $B P$ blood pressure, $C O$ cardiac output, $P C A$ pulse contour analysis, ICU intensive care unit, SVV stroke volume variation, $P P V$ pulse pressure variation, GEDV global end diastolic volume, EVLW extravascular lung water, SVR systemic vascular resistance, CO cardiac output, AR aortic regurgitation, NDM non-depolarizing muscle relaxant, $P A C$ pulmonary artery catheter 


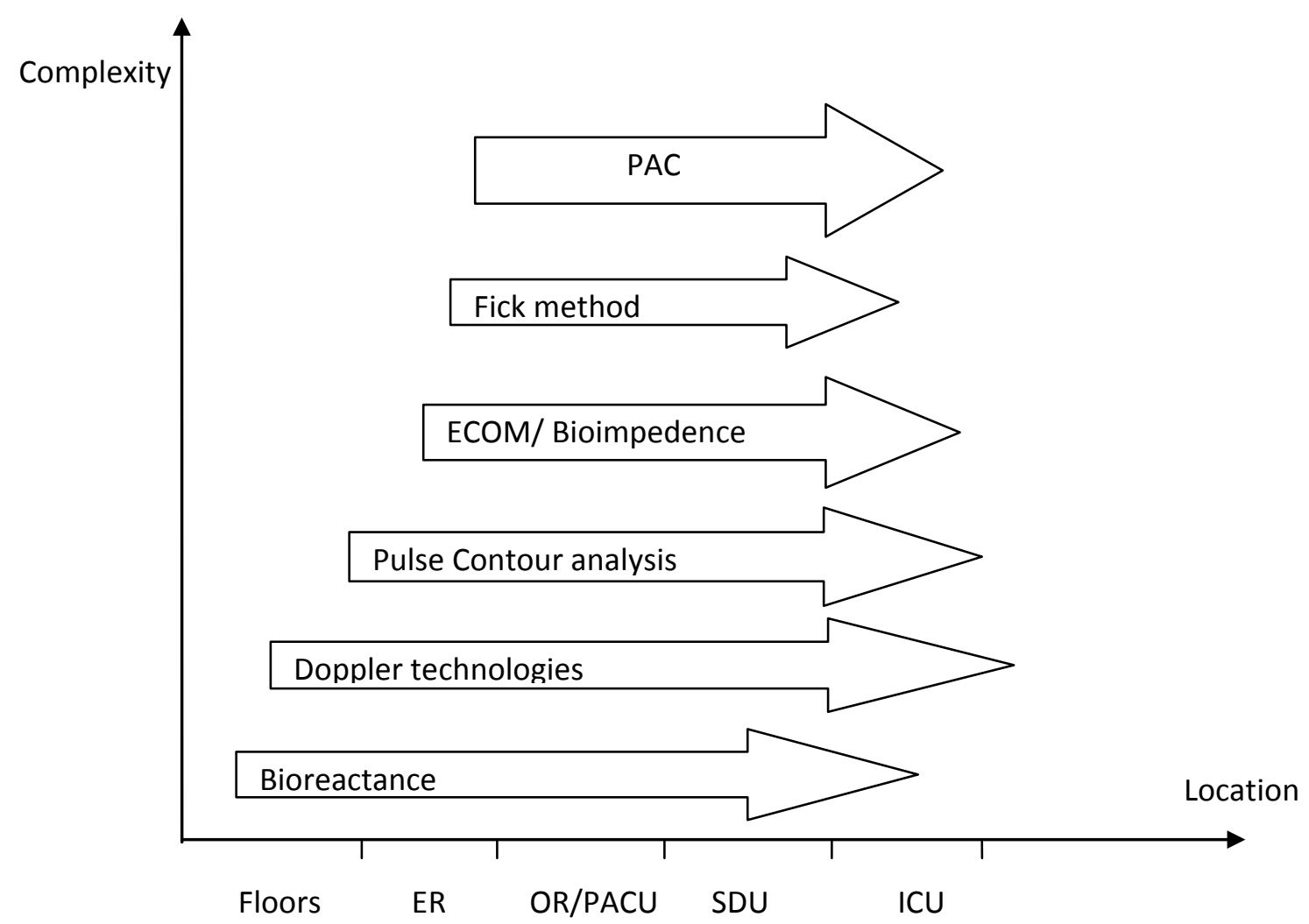

Figure 4 Suggested use of cardiac output devices and monitoring system within the hospital system. $E R=E m e r g e n c y$ room, $O R / P A C U=$ operating room/post-anesthesia care unit, SDU = step down unit, ICU = intensive care unit, ECOM = endotracheal cardiac output monitoring.

precision of this device in acute lung injury since the shunt fraction is estimated from the concentration of the inspired fraction of oxygen and arterial oxygen saturation. Its main area of use has been in stable cardiac patients. This device can be utilized in the busy emergency medicine environment for evaluation of low cardiac output states if a patient is already intubated and on mechanical ventilation.

\section{Conclusions}

With an increasing need for utilization of hemodynamic monitoring due to the aging population, increased comorbidities and increasingly complex interventions and monitoring are becoming incorporated into the standard of care, and the need for hemodynamic monitoring is likely to increase. Because of the inherent limitations and complications of PAC in the busy emergency department, physicians are looking for less invasive devices to measure $\mathrm{CO}$. There is no gold standard for the clinical measurement of CO. Therefore, comparison of these new technologies is somewhat challenging (Table 1). The level of invasiveness and complexity may help the clinicians decide where to best use the devices (Figure 4).

\section{Acknowledgements}

JP, GZ, SC, and NN contributed towards the article by making substantial contributions to conception, design, acquisition of data, or analysis and interpretation of data and were involved in drafting the manuscript or revising it critically for important intellectual content. No funding was provided for the manuscript preparation.

\section{Author details}

${ }^{1}$ VA Western New York Healthcare System, Division of Critical Care and Pain Medicine, Department of Anesthesiology, State University of New York at Buffalo School of Medicine and Biomedical Sciences, Buffalo, NY, USA ${ }^{2}$ University of lowa, Mason City Cardiology, Mason City, lowa, USA ${ }^{3}$ VA Medical Center, Rm 203C, 3495 Bailey Ave, Buffalo, NY 14215, USA

\section{Authors' contributions}

JP drafted the manuscript. GZ drafted the manuscript and made significant contributions to the revision. SC drafted the manuscript and made significant contributions to the revision. NN drafted the manuscript and provided figures. All authors read and approved the final manuscript.

\section{Competing interests}

The authors do not have any financial and personal relationships with other people or organizations that could inappropriately influence (bias) their work. Examples of potential conflicts of interest include employment, consultancies, stock ownership, honoraria, paid expert testimony, patent applications/registrations, and grants or other funding.

Received: 27 September 2010 Accepted: 24 April 2012 Published: 24 April 2012 


\section{References}

1. Fegler G: Measurement of cardiac output in anaesthetized animals by a thermodilution method. Q J Exp Physiol Cogn Med Sci 1954, 39(3):153-164

2. Stewart GN: Researches on the circulation time and on the influences which affect it. J Physiol 1897, 22(3):159-183.

3. Reade MC, Angus DC: PAC-Man: game over for the pulmonary artery catheter? Crit Care 2006, 10(1):303.

4. Ivanov R, Allen J, Calvin JE: The incidence of major morbidity in critically ill patients managed with pulmonary artery catheters: a meta-analysis. Crit Care Med 2000, 28(3):615-619.

5. Kahwash R, Leier CV, Miller L: Role of the pulmonary artery catheter in diagnosis and management of heart failure. Heart Fail Clin 2009, 5(2):241-248

6. Shah MR, et al: Evaluation Study of Congestive Heart Failure and Pulmonary Artery Catheterization Effectiveness (ESCAPE): design and rationale. Am Heart J 2001, 141(4):528-535.

7. Side CD, Gosling RG: Non-surgical assessment of cardiac function. Nature 1971, 232(5309):335-336.

8. Dark PM, Singer M: The validity of trans-esophageal Doppler ultrasonography as a measure of cardiac output in critically ill adults. Intensive Care Med 2004, 30(11):2060-2066.

9. Singer M, Bennett ED: Noninvasive optimization of left ventricular filling using esophageal Doppler. Crit Care Med 1991, 19(9):1132-1137.

10. Madan AK, et al: Esophageal Doppler ultrasound monitor versus pulmonary artery catheter in the hemodynamic management of critically ill surgical patients. J Trauma 1999, 46(4):607-611, discussion 611612.

11. Isakow W, Schuster DP: Extravascular lung water measurements and hemodynamic monitoring in the critically ill: bedside alternatives to the pulmonary artery catheter. Am J Physiol Lung Cell Mol Physiol 2006, 291(6): L1118-L1131.

12. Rodriguez RM, Berumen KA: Cardiac output measurement with an esophageal doppler in critically ill Emergency Department patients. $J$ Emerg Med 2000, 18(2):159-164.

13. Marquez J, et al: Ability of pulse power, esophageal Doppler, and arterial pulse pressure to estimate rapid changes in stroke volume in humans. Crit Care Med 2008, 36(11):3001-3007.

14. Seoudi HM, et al: The esophageal Doppler monitor in mechanically ventilated surgical patients: does it work? J Trauma 2003, 55(4):720-725, discussion 725-726.

15. Atlas GM: Development and application of a logistic-based systolic model for hemodynamic measurements using the esophageal Doppler monitor. Cardiovasc Eng 2008, 8(3):159-173.

16. Dabrowski GP, et al: A critical assessment of endpoints of shock resuscitation. Surg Clin North Am 2000, 80(3):825-844.

17. Marik PE: Pulmonary artery catheterization and esophageal doppler monitoring in the ICU. Chest 1999, 116(4):1085-1091.

18. Royse CF: Ultrasound-guided haemodynamic state assessment. Best Pract Res Clin Anaesthesiol 2009, 23(3):273-283.

19. Price $S$, Uddin S, Quinn T: Echocardiography in cardiac arrest. Curr Opin Crit Care 2010, 16(3):211-215.

20. Mookadam F, et al: Critical appraisal on the utility of echocardiography in the management of acute pulmonary embolism. Cardiol Rev 2010, 18(1):29-37.

21. Breitkreutz R, Walcher F, Seeger FH: Focused echocardiographic evaluation in resuscitation management: concept of an advanced life supportconformed algorithm. Crit Care Med 2007, 35(5 Suppl):S150-S161.

22. Parato VM, et al: Resting echocardiography for the early detection of acute coronary syndromes in chest pain unit patients. Echocardiography 2010, 27(6):597-602.

23. Longjohn $\mathrm{M}$, et al: Point-of-care echocardiography by pediatric emergency physicians. Pediatr Emerg Care 2011, 27(8):693-696.

24. Randazzo MR, et al: Accuracy of emergency physician assessment of left ventricular ejection fraction and central venous pressure using echocardiography. Acad Emerg Med 2003, 10(9):973-977.

25. Wesseling $\mathrm{KH}$, et al: A computer module for the continuous monitoring of cardiac output in the operating theatre and the ICU. Acta Anaesthesiol Belg 1976, 27(suppl):327-341.

26. Eisenberg PR, et al: A prospective study of lung water measurements during patient management in an intensive care unit. Am Rev Respir Dis 1987, 136(3):662-668
27. Della Rocca G, et al: Cardiac output monitoring: aortic transpulmonary thermodilution and pulse contour analysis agree with standard thermodilution methods in patients undergoing lung transplantation. Can J Anaesth 2003, 50(7):707-711.

28. Halvorsen PS, et al: Agreement between PiCCO pulse-contour analysis, pulmonal artery thermodilution and transthoracic thermodilution during off-pump coronary artery by-pass surgery. Acta Anaesthesiol Scand 2006 50(9):1050-1057

29. Cottis R, Magee N, Higgins DJ: Haemodynamic monitoring with pulseinduced contour cardiac output (PiCCO) in critical care. Intensive Crit Care Nurs 2003, 19(5):301-307.

30. Hung MH, et al: Application of Pulse Contour Cardiac Output (PiCCO) system for adequate fluid management in a patient with severe reexpansion pulmonary edema. Acta Anaesthesiol Taiwan 2008, 46(4):187-190.

31. Aguilar G, Belda FJ, Perel A: Minimally invasive cardiopulmonary monitoring with the PiCCO Plus system. Rev Esp Anestesiol Reanim 2008, 55(2):90-100.

32. Uchino $\mathrm{S}$, et al: Pulmonary artery catheter versus pulse contour analysis: a prospective epidemiological study. Crit Care 2006, 10(6):R174.

33. Mehta $Y$, et al: Cardiac output monitoring: comparison of a new arterial pressure waveform analysis to the bolus thermodilution technique in patients undergoing off-pump coronary artery bypass surgery. $J$ Cardiothorac Vasc Anesth 2008, 22(3):394-399.

34. Mayer J, et al: Cardiac output derived from arterial pressure waveform analysis in patients undergoing cardiac surgery: validity of a second generation device. Anesth Analg 2008, 106(3):867-872, table of contents.

35. Scheeren TW, et al: Performance of a minimally invasive cardiac output monitoring system (Flotrac/Vigileo). Br J Anaesth 2008, 101(2):279-280.

36. Langewouters GJ, Wesseling KH, Goedhard WJ: The pressure dependent dynamic elasticity of 35 thoracic and 16 abdominal human aortas in vitro described by a five component model. J Biomech 1985 , 18(8):613-620.

37. Button D, et al: Clinical evaluation of the FloTrac/Vigileo system and two established continuous cardiac output monitoring devices in patients undergoing cardiac surgery. Br J Anaesth 2007, 99(3):329-336.

38. McLean AS, et al: Comparison of cardiac output measurements in critically ill patients: FloTrac/Vigileo vs transthoracic Doppler echocardiography. Anaesth Intensive Care 2011, 39(4):590-598.

39. Reid RD, Jayamaha J: The use of a cardiac output monitor to guide the initial fluid resuscitation in a patient with burns. Emerg Med J 2007, 24(5): e32

40. Sotomi $Y$, et al: Reliability of a less-invasive cardiac output monitor in the perioperative period of a patient with severe dilated cardiomyopathy. Masui 2011, 60(2):203-207.

41. Linton RA, Band DM, Haire KM: A new method of measuring cardiac output in man using lithium dilution. British J Anaesthesia 1993, 71(2):262-266

42. Pearse RM, Ikram K, Barry J: Equipment review: an appraisal of the LiDCO plus method of measuring cardiac output. Crit Care 2004, 8(3):190-195.

43. Kurita $T$, et al: Comparison of the accuracy of the lithium dilution technique with the thermodilution technique for measurement of cardiac output. British J Anaesthesia 1997, 79(6):770-775.

44. Costa MG, et al: Continuous and intermittent cardiac output measurement in hyperdynamic conditions: pulmonary artery catheter vs. lithium dilution technique. Intensive Care Med 2008, 34(2):257-263.

45. Kubicek WG, et al: Development and evaluation of an impedance cardiac output system. Aerosp Med 1966, 37(12):1208-1212.

46. Summers RL, et al: Bench to bedside: electrophysiologic and clinical principles of noninvasive hemodynamic monitoring using impedance cardiography. Acad Emerg Med 2003, 10(6):669-680.

47. Gujjar AR, et al: Non-invasive cardiac output by transthoracic electrical bioimpedence in post-cardiac surgery patients: comparison with thermodilution method. J Clin Monit Comput 2008, 22(3):175-180.

48. Van De Water JM, et al: Impedance cardiography: the next vital sign technology? Chest 2003, 123(6):2028-2033.

49. Maus TM, et al: Cardiac output determination from endotracheally measured impedance cardiography: clinical evaluation of endotracheal cardiac output monitor. J Cardiothorac Vasc Anesth 2011, 25(5):770-775. 
50. Fellahi $\mathrm{JL}$, et al: A comparison of endotracheal bioimpedance cardiography and transpulmonary thermodilution in cardiac surgery patients. J Cardiothorac Vasc Anesth 2012, 26(2):217-222.

51. Wallace AW, et al: Endotracheal cardiac output monitor. Anesthesiology 2000, 92(1):178-189.

52. Kossari N, Hufnagel G, Squara P: Bioreactance: a new tool for cardiac output and thoracic fluid content monitoring during hemodialysis. Hemodial Int 2009, 13(4):512-517.

53. Benomar $B$, et al: Fluid responsiveness predicted by noninvasive bioreactance-based passive leg raise test. Intensive Care Med 2010 36(11):1875-1881.

54. Raval NY, et al: Multicenter evaluation of noninvasive cardiac output measurement by bioreactance technique. J Clin Monit Comput 2008, 22(2):113-119.

55. Ohashi $Y$, et al: Non-invasive hemodynamic assessment of non-pregnant, healthy pregnant and preeclamptic women using bioreactance. [corrected]. Revista Brasileira De Anestesiologia 2010, 60(6):603-613, 335-340.

56. Squara $P$, et al: Comparison of monitoring performance of Bioreactance vs. pulse contour during lung recruitment maneuvers. Crit Care 2009, 13(4):R125.

57. Karpman $\mathrm{VL}$ : The theoretical analysis of Fick's equation. On the centennial of the use of Fick's principle in physiology. Z Kardiol 1975, 64(9):801-808.

58. Shepherd AP, et al: Local control of tissue oxygen delivery and its contribution to the regulation of cardiac output. Am J Physiol 1973, 225(3):747-755.

59. Ehlers KC, et al: Cardiac output measurements. A review of current techniques and research. Ann Biomed Eng 1986, 14(3):219-239.

60. de Abreu MG, et al: Partial carbon dioxide rebreathing: a reliable technique for noninvasive measurement of nonshunted pulmonary capillary blood flow. Crit Care Med 1997, 25(4):675-683.

61. Green DW: Comparison of cardiac outputs during major surgery using the Deltex CardioQ oesophageal Doppler monitor and the NovametrixRespironics NICO: a prospective observational study. Int I Surg 2007, 5(3):176-182.

doi:10.1186/1865-1380-5-18

Cite this article as: Porhomayon et al:: Applications of minimally invasive cardiac output monitors. International Journal of Emergency Medicine 2012 5:18

\section{Submit your manuscript to a SpringerOpen ${ }^{\circ}$ journal and benefit from:}

- Convenient online submission

- Rigorous peer review

- Immediate publication on acceptance

- Open access: articles freely available online

- High visibility within the field

- Retaining the copyright to your article

Submit your next manuscript at $\gg$ springeropen.com 